\title{
Relación neutrófilo a linfocito, recuento linfocitario e índices de actividad inflamatoria como factores pronóstico en infecciones por SARS-CoV-2: Un estudio de cohorte prospectiva
}

\author{
Neutrophil to lymphocite ratio and inflammatory biomarkers as prognostic \\ factors amongst patients with COVID-19: A prospective cohort study
}

Felipe Martínez MD. MSC ${ }^{1,2,{ }^{*},}$ Dominique Boisier MD. ${ }^{1}$, Constanza Vergara MD. ${ }^{1}$, Jaime Vidal MD. ${ }^{1}$

Unidad de Cuidados Intensivos, Hospital Naval Almirante Nef, Viña del Mar.

2 Escuela de Medicina, Universidad Andrés Bello, Sede Viña del Mar, Viña del Mar.

Conflictos de Interés: Los autores declaran no tener ningún conflicto de interés que declarar.

Financiamiento: Este estudio fue realizado en forma independiente, sin recibirse fondos para su ejecución.

Fecha de recepción: 30 de junio de 2021 / Fecha de aceptación: 18 de julio de 2021

\begin{abstract}
Introduction: Patients with COVID-19 can develop respiratory failure requiring treatment with invasive mechanical ventilation (IMV) and death. It is important to have clinical predictors of these outcomes. Objetives: To establish the diagnostic accuracy of neutrophil to lymphocyte ratios (NLR) in predicting the need of IMV and survival amongst patients with COVID-19 and to compare this accuracy with other laboratory tests. Methodology: Prospective cohort study of hospitalized patients with a SARS-CoV-2 infection confirmed by RT-PCR. Clinical, demographic and laboratory predictors were assessed, including LDH, C-reactive protein, absolute lymphocyte counts, serum ferritin and NLR. Statistical analyses were undertaken using receiver-operator characteristics (ROC) curves, which were in turn compared using the method described by Hanley and McNeal. Results: One hundred and twelve patients were studied, most were male (60.7\%) with a mean age of $63.4 \pm 18.3$ years. Twenty-two patients required IMV during their stay and 28 died. The NLR showed a good diagnostic accuracy in detecting patients that would require IMV (AUC $0.70,95 \% \mathrm{Cl} 0.57-0.86$ ) or died during the hospitalization (AUC $0.83,95 \% \mathrm{Cl} 0.75-0.91$ ). A cutoff point of 5.5 or higher had an $80.8 \%$ sensitivity and $73.1 \%$ specificity in detecting patients that died during their stay. Conclusions. NLR showed favorable diagnostic properties in detecting patients with COVID-19 at risk of adverse outcomes. Its wide availability and low cost are desirable features that might facilitate its implementation in routine clinical practice.
\end{abstract}

Key words: SARS-CoV-2, COVID-19, neutrophils, lymphocytes, prognosis, mechanical ventilation, survival.

\section{RESUMEN}

Introducción: La infección a SARS-CoV-2 puede generar insuficiencia respiratoria con requerimientos de ventilación mecánica invasiva (VMI). Contar con predictores de este evento es fundamental. Objetivos: Determinar la capacidad diagnóstica de la relación neutrófilo a linfocito (RNL) para predecir necesidad de VMI o muerte y compararla con otros índices de laboratorio. Métodos: Cohorte prospectiva de pacientes hospitalizados con diagnóstico confirmado de COVID-19 por PCR. Se evaluaron predictores clínicos, demográficos y de laboratorio, incluyendo LDH, proteína C reactiva, recuento linfocitario, ferritina y la RNL. Los pacientes fueron seguidos hasta el alta hospitalaria. El análisis estadístico se realizó mediante curvas de características de receptor-operador (ROC) que se compararon mediante el método de Hanley y McNeal. Resultados: Se estudiaron 112 pacientes, $60,7 \%$ hombres con media de edad de $63,4 \pm 18,3$ años. Veintidos ingresaron a ventilación invasiva y 28 fallecieron. La RNL mostró una buena capacidad diagnóstica para detectar pacientes que necesitaron VMI (AUC: 0,70, IC95\% 0,57-0,86) o falle-

felipe.martinez.l@unab.cl

*ORCID: https://orcid.org/0000-0002-9857-8403 
cieron durante la hospitalización (AUC: 0,83, IC95\% 0,75-0,91). Un punto de corte de 5,5 o superior tuvo una sensibilidad del $80,8 \%$ y especificidad del $73,1 \%$ para detectar pacientes en riesgo de fallecer por la enfermedad. Conclusiones: La RNL tiene favorables aptitudes diagnósticas en establecer el riesgo de evolución tórpida en casos de infecciones a SARS-CoV-2. La amplia disponibilidad de la prueba y su bajo costo representan características atractivas para su uso en clínica.

Palabras clave: SARS-CoV-2, COVID-19, neutrófilos, linfocitos, pronóstico, ventilación mecánica, supervivencia.

\section{Introducción}

A fines de 2019, un nuevo coronavirus (SARS-CoV-2) fue identificado en China como agente causal de una nueva enfermedad respiratoria Ilamada COVID-19. Desde entonces, el virus se ha convertido en una emergencia de salud global, afectando más de 200 países, con más de 10 millones de infectados y más 500.000 muertes asociadas a la enferme$\operatorname{dad}[1],[2]$

El virus se transmite principalmente a través de exposición a gotitas, ingresando a la célula mediante la unión de la proteína $\mathrm{S}$ (spike) al receptor de angiotensina 2 (ACE2), que se localiza en el epitelio nasal, bronquial y neumocitos. La proteasa transmembrana (TMPRSS2), presente en la célula huésped, promueve el ingreso del coronavirus a través de la activación de la proteína S y la escisión del receptor ACE2[1]. Posterior a la endocitosis, el virus utiliza la maquinaria celular para replicarse y liberar más viriones a circulación, permitiendo la propagación de la infección. En estadios iniciales, las células infectadas liberan moléculas pro-inflamatorias que permiten reclutar linfocitos $T$, monocitos y neutrófilos. La respuesta inflamatoria viral innata y adaptativa, altera la linfopoyesis y aumenta la apoptosis linfocitaria. Esto sumado a la posibilidad de que el virus infecte directamente el linfocito $T$, contribuye a generar linfopenia, similar a otros escenarios de enfermedades virales[3]. En etapas posteriores de la enfermedad, se acelera la replicación viral, se altera la barrera epitelial y conforme aumenta la respuesta inflamatoria, ingresan monocitos y neutrófilos a la célula endotelial y espacio aéreo. Esta inflamación mononuclear intersticial y edema, se traduce en el desarrollo de falla respiratoria[4].

El período promedio de incubación es de 5 días (2-7). Al cabo de este tiempo, la gran mayoría de los pacientes desarrollará síntomas leves, tales como tos, cefalea, fiebre, mialgias, diarrea, o permanecerá asintomático[5]-[7]. Mientras que una minoría se presentará con neumonía grave, síndrome de distress respiratorio del adulto, falla multiorgánica y muerte. La proporción de pacientes que requieren cuidados intensivos por estos motivos es variable en la literatura, pero un rango entre $2 \%$ a $10 \%$ ha sido frecuentemente reportado[5],[6],[8].

La mortalidad hospitalaria general se describe entre 15\% a $20 \%$ y aumenta sobre $40 \%$ en los pacientes que requieren ingreso a Unidades de Cuidado Intensivo. Al evaluar la mortalidad hospitalaria según grupo etario, esta se incrementa a mayor edad, siendo 2 a 3 veces superior en pacientes sobre 70 años[9],[10]. Dentro de las alteraciones de laboratorio características de los sujetos hospitalizados, se describen linfopenia (recuento absoluto de linfocitos $<1.000$ células/uL), aumento de los parámetros de actividad inflamatoria (proteína $C$ reactiva, ferritina, IL-6), aumento de lactato deshidrogenasa y pa- rámetros de coagulación anormales (elevación de dímero $D$, trombocitopenia, prolongación del tiempo de protrombina). Sin embargo, la mayoría de estas alteraciones son inespecíficas y pueden presentarse en neumonía por otras causas.

El presente estudio pretende evaluar a exámenes de laboratorio general como potenciales factores pronóstico, capaces de clasificar oportunamente a los enfermos de mayor gravedad. De esta forma poder disponer de una herramienta sencilla que permita preparar con anticipación el soporte adecuado y orientar de manera óptima la distribución de recursos.

\section{Metodología}

Con el fin de determinar la capacidad diagnóstica del recuento linfocitario y otros índices de laboratorio en predecir desenlaces entre pacientes hospitalizados por SARS-CoV-2, se realizará un estudio de cohorte prospectiva entre pacientes hospitalizados entre los meses de marzo y junio de 2020 en el Hospital Naval Almirante Nef. El presente protocolo ha sido redactado en concordancia con las pautas Strengthening the Reporting of Observational Studies in Epidemiology (STROBE) en su versión para estudios de cohorte[11]. El Comité de Ética del Hospital Naval Almirante Nef revisó y aprobó la ejecución de este estudio.

\section{Participantes}

Se realizó un muestreo consecutivo de pacientes adultos (> 18 años de edad) con diagnóstico confirmado de neumonía a SARS-CoV-2 mediante una prueba RT-PCR que consultaron en el Servicio de Urgencias del Hospital Naval Almirante Nef. Fueron excluidos del estudio los pacientes trasladados de otro centro asistencial del estudio para minimizar sesgos secundarios a diferencias en registro y variabilidad en técnicas de laboratorio en el análisis.

Se registraron datos básicos en todos los pacientes que ingresaron al estudio. Estos datos incluyeron aspectos demográficos como sexo y edad, fecha de inicio de síntomas asociados a la infección al nuevo coronavirus y comorbilidades relevantes en base a lo establecido por el Índice de Comorbilidades de Charlson[12],[13]. Asimismo se estableció la severidad de la enfermedad en base a la escala SOFA medida al ingreso en el Servicio de Urgencias. Esta escala ha sido seleccionada por sus apropiadas capacidades diagnósticas, así como lo común de su uso en Chile y otros países del mundo[14]-[16]. También se consideraron los exámenes de laboratorio pertinentes para el cálculo del SOFA como datos individuales, niveles de proteína $C$ reactiva y la lactacidemia de ingreso al hospital. Toda la información fue registrada en un formulario especialmente diseña- 
do para ello utilizando códigos que impidieran la identificación posterior de pacientes individuales.

\section{Variables}

Fuera del perfil epidemiológico y de laboratorio arriba indicado, las variables de exposición del presente estudio incluyeron al recuento linfocitario absoluto, relación neutrófilo: linfocito, proteína $C$ reactiva, lactato deshidrogenasa y ferritina sérica, todos medidos al ingreso. Los recuentos hematológicos fueron obtenidos a partir del contador hematológico Beckman Coulter DxH800 ${ }^{\circledR}$ (Beckman Coulter Diagnostics, California, Estados Unidos), mientras que los niveles de lactato deshidrogenasa se evaluaron a través de la reacción de lactato a piruvato mediante un analizador Alinity- $c^{\circledR}$ (Abbott Core Laboratory, Abbott Diagnostics, Illinois, Estados Unidos) usando estándares de la Federación Internacional de Química Clínica (IFCC). La ferritina sérica fue estimada utilizando un equipo Alinity-i ${ }^{\circledR}$ (Abbott Core Laboratory, Abbott Diagnostics, Illinois, Estados Unidos) mediante inmunoanálisis quimioluminiscente de micropartículas (CMIA).

\section{Desenlaces}

El desenlace primario del presente trabajo es la supervivencia al episodio de COVID-19. Como desenlaces secundarios se consideró al ingreso a la Unidad de Cuidados Intensivos (UCI) para iniciar ventilación mecánica invasiva. Para determinar estos desenlaces se siguió a todos los pacientes incluidos hasta su alta hospitalaria.

\section{Análisis Estadístico}

\section{Tamaño muestral}

Literatura previa ha descrito a la linfocitopenia como uno de los hallazgos sugerentes de una enfermedad COVID-19 severa, siendo aparentemente un fenómeno más común entre pacientes críticos. Basado en varios reportes[3],[7],[17],[18], se estimó que un total de 100 pacientes serían necesarios para obtener una potencia estadística del $90 \%$ en demostrar diferencia media absoluta de 200 linfocitos entre pacientes que sobreviven a la infección versus quienes no (1.000 vs 800 linfocitos), asumiendo una desviación estándar común de dichas medias de 300 células a niveles de significancia estándar (alfa de 5\% a dos colas). Adicionalmente, se calculó que 90 pacientes serían necesarios para obtener $85 \%$ de potencia estadística en detectar un área bajo la curva ROC teórica de 0,75 para la relación de neutrófilo a linfocito, asumiendo una tasa de mortalidad intrahospitalaria del 10\%. En base a estos resultados, se estableció un tamaño muestral mínimo para llevar a cabo el estudio de 100 pacientes, que se incrementó un $10 \%$ para dar cuenta de potenciales pérdidas de seguimiento.

\section{Estrategia de análisis}

Para el análisis estadístico se utilizaron medias, desviación estándar, frecuencias absolutas y relativas para el análisis descriptivo. Para el análisis inferencial se emplearon las pruebas $t$ de Student o de Mann Whitney para la comparación de medias según las características de distribución y varianzas de los datos obtenidos. Eventuales asociaciones entre variables cua- litativas fueron exploradas mediante el estadístico de Fisher, mientras que asociaciones lineales entre variables cuantitativas se estudiaron empleando la prueba de Pearson o el estadístico de Kendall según la distribución de estos datos cuantitativos. Asimismo se calcularon intervalos de confianza del 95\% para estimadores puntales siempre que esto fue pertinente.

La capacidad diagnóstica de las pruebas de laboratorio seleccionadas fue evaluada mediante la aplicación de curvas de características de receptor-operador (ROC). Se cuantificó la capacidad predictiva de estas pruebas a través del área bajo la curva (AUC) asociado a sus correspondientes intervalos de confianza del $95 \%$ y se estableció un punto de corte en base a la mejor capacidad predictiva posible en base a estas curvas. Las comparaciones entre pruebas de laboratorio se llevaron a cabo utilizando el método de Hanley y McNeal.

La asociación de alteraciones del laboratorio con el pronóstico de pacientes hospitalizados fue también cuantificada mediante curvas de supervivencia de Kaplan-Meier. Dichas curvas fueron a su vez comparadas mediante el estadístico logrank o alguna de sus variaciones en función de las características de la función, como el de Gehan y Breslow o el estadístico de Peto-Prentice. Se cuantificaron asociaciones mediante la función de Hazard ratio con su correspondiente intervalo de confianza del 95\%. Todos los análisis fueron realizados por un estadístico independiente del proceso de evaluación de pacientes utilizando datos anonimizados en el software STATA 16.0 (SE) para MacOs.

\section{Resultados}

\section{Características de los pacientes}

Durante el período de estudio se hospitalizaron 112 pacientes con diagnóstico confirmado de COVID-19 en el Hospital Naval Almirante Nef. La mayoría fueron hombres (68 pacientes, $60,7 \%)$, con una edad promedio de 63,4 $\pm 18,3$ años. La media de síntomas al ingreso fue de 6 días (rango intercuartil, RIC 2 a 8 días). El 43\% de los pacientes tenía historia de hipertensión, $27,8 \%$ eran diabéticos y $9,2 \%$ de los pacientes tenía historia de insuficiencia cardiaca. El puntaje mediana del índice de comorbilidades de Charlson fue de 2 puntos (RIC 0-5 puntos).

En general, la mayoría de los pacientes no mostró una gran disfunción de órganos más alla de lo respiratorio, con un puntaje mediana en la escala SOFA de 1 (RIC: 0-2) puntos al momento de su ingreso al hospital. La media de Pa/Fi al ingreso fue de $305 \pm 114$, mientras que la lactacidemia promedio de ingreso fue 10,3 $\pm 6,2 \mathrm{mg} / \mathrm{dL}$ y sólo 5 enfermos se presentaron con cifras de lactacidemia mayores a $20 \mathrm{mg} / \mathrm{dL}$.

La mayoría de los pacientes presentó elevaciones de índices de actividad inflamatoria al ingreso del hospital. La proteína C reactiva promedio fue de 90,0 $\pm 81,2 \mathrm{mg} / \mathrm{Lt}$, mientras que el hemograma mostró un recuento leucocitario promedio de $7.500 \pm 3.050$ células entre los pacientes ingresados. El número medio de neutrófilos fue de $5.500 \pm 3.000$ células en ambos grupos, mientras que el de linfocitos fue de $1.350 \pm$ 1.056 células. También se apreciaron elevaciones importantes en la ferritina de ingreso de los pacientes estudiados, con una cifra media de $1.431 \pm 2.440 \mathrm{ug} / \mathrm{dL}$, la que se acompañó de elevaciones de lactato deshidrogenasa a $332 \pm 160$ UI/L. Se muestra un resumen de las principales características clínicas y de laboratorio de la cohorte en la Tabla 1. 


\begin{tabular}{|c|c|c|c|c|}
\hline Característica & $\begin{array}{l}\text { Sobrevivientes } \\
\quad n=84\end{array}$ & $\begin{array}{l}\text { Fallecidos } \\
n=28\end{array}$ & $\begin{array}{l}\text { Todos } \\
n=112\end{array}$ & Valor $p$ \\
\hline \multicolumn{5}{|l|}{ Aspectos clínicos } \\
\hline Sexo masculino (n, \%) & $51(61,9 \%)$ & $16(57,1 \%)$ & $68(60,7 \%)$ & 0,66 \\
\hline Edad (años) (media, DE) & $57,3 \pm 16,1$ & $81,7 \pm 10,6$ & $63,4 \pm 18,3$ & $<0,001$ \\
\hline $\begin{array}{l}\text { Mediana de tiempo de síntomas al } \\
\text { ingreso (RIC) }\end{array}$ & $6,5(3,5-10)$ & $3,0(0,5-6,5)$ & $6(2-8)$ & 0,007 \\
\hline Diabetes mellitus ( $\mathrm{n}, \%$ ) & $24(28,9 \%)$ & $7(25 \%)$ & $31(27,9 \%)$ & 0,81 \\
\hline Hipertensión arterial (n, \%) & $28(33,7 \%)$ & $20(71,4 \%)$ & $48(43,2 \%)$ & 0,001 \\
\hline $\begin{array}{l}\text { Enfermedad pulmonar obstructiva crónica } \\
(\mathrm{n}, \%)\end{array}$ & $2(2,4 \%)$ & $0(0 \%)$ & $2(1,8 \%)$ & $>0,99$ \\
\hline Cáncer previo (n, \%) & $6(7,1 \%)$ & $3(11,1 \%)$ & $9(8,1 \%)$ & 0,69 \\
\hline Insuficiencia cardiaca (n, \%) & $4(4,9 \%)$ & $6(21,4 \%)$ & $10(9,2 \%)$ & 0,02 \\
\hline Enfermedad renal crónica (n, \%) & $6(7,1 \%)$ & $1(3,6 \%)$ & $7(6,3 \%)$ & 0,68 \\
\hline $\begin{array}{l}\text { Mediana del índice de comorbilidades de } \\
\text { Charlson (RIC) }\end{array}$ & $2(0-3)$ & $6(3,5-6)$ & $2(0-5)$ & $<0,001$ \\
\hline \multicolumn{5}{|l|}{ Laboratorio de Ingreso } \\
\hline Media de $\mathrm{pH}(\mathrm{DE})(\mathrm{mmHg})$ & $7,44 \pm 0,06$ & $7,41 \pm 0,11$ & $7,43 \pm 0,07$ & 0,08 \\
\hline Media de Pa/Fi (DE) (mmHg) & $332 \pm 102$ & $209 \pm 104$ & $305 \pm 114$ & $<0,001$ \\
\hline Media de $\mathrm{PCO}_{2}(\mathrm{DE})(\mathrm{mmHg})$ & $33,5 \pm 7,7$ & $34,7 \pm 10,1$ & $33,8 \pm 8,2$ & 0,60 \\
\hline Media de bicarbonato (DE) (mEq/Lt) & $22,1 \pm 3,0$ & $20,9 \pm 4,0$ & $21 \pm 9 \pm 3,3$ & 0,14 \\
\hline Media de hemoglobina (DE) (g/dL) & $13,9 \pm 2,2$ & $12,4 \pm 2,0$ & $13,5 \pm 2,3$ & 0,003 \\
\hline Media de leucocitos (DE) (cels/mm³) & $6.920 \pm 2.540$ & $9.300 \pm 3.800$ & $7.500 \pm 3050$ & $<0,001$ \\
\hline Media de neutrófilos (DE) (cels/mm³) & $4.710 \pm 2.280$ & $7.900 \pm 3.600$ & $5.500 \pm 3.000$ & $<0,001$ \\
\hline Media de linfocitos (DE) (cels/mm³) & $1.510 \pm 1.155$ & $855 \pm 370$ & $1.350 \pm 1.056$ & 0,005 \\
\hline Media de plaquetas (DE) $\left(1.000\right.$ cels $\left./ \mathrm{mm}^{3}\right)$ & $241 \pm 78$ & $226 \pm 61$ & $237 \pm 74$ & 0,38 \\
\hline Media de proteína $C$ reactiva $(D E)(m g / L t)$ & $75,2 \pm 72,3$ & $136,5 \pm 91,1$ & $90,0 \pm 81,2$ & $<0,001$ \\
\hline $\begin{array}{l}\text { Media de lactato deshidrogenasa (DE) } \\
(\mathrm{UI} / \mathrm{dL})\end{array}$ & $306 \pm 34$ & $427 \pm 205$ & $332 \pm 160$ & 0,002 \\
\hline Media de ferritina (DE) (ug/dL) & $1.173 \pm 1.432$ & $2.325 \pm 4.395$ & $1.431 \pm 2.440$ & 0,07 \\
\hline Media de creatininemia (DE) (mg/dL) & $1,25 \pm 1,4$ & $2,0 \pm 2,2$ & $1,43 \pm 1,65$ & 0,06 \\
\hline Media de uremia (DE) (mg/dL) & $39,6 \pm 36,0$ & $86,4 \pm 59,5$ & $50,9 \pm 47,1$ & $<0,001$ \\
\hline Media de lactacidemia (DE) (mg/dL) & $9,70 \pm 4,3$ & $12,3 \pm 10,0$ & $10,3 \pm 6,2$ & 0,10 \\
\hline Media de sodio (DE) (mEq/Lt) & $137 \pm 3,7$ & $138 \pm 6,5$ & $138 \pm 4,5$ & 0,54 \\
\hline Media de potasio (DE) (mEq/Lt) & $4,2 \pm 0,46$ & $4,3 \pm 1,0$ & $4,2 \pm 0,64$ & 0,39 \\
\hline Media de cloro (DE) (mEq/Lt) & $103 \pm 4,5$ & $104 \pm 6,6$ & $104 \pm 5,1$ & 0,44 \\
\hline Media de puntaje SOFA (RIC) & $1(0-2)$ & $2(2-4)$ & $1(0-2)$ & $<0,001$ \\
\hline
\end{tabular}

\section{Desenlaces}

Treinta y cuatro pacientes (30,4\%; IC95\% 22\%-39,8\%) requirieron ingreso a $\mathrm{UCI}$ para manejo de la insuficiencia respiratoria. Veintidos (65\%) fueron conectados a ventilación mecánica invasiva. La estadía mediana en la UCI fue de 10 (RIC 6-20 días) y 9 de los pacientes ingresados a la UCI fallecieron durante la hospitalización (26,5\%, IC 95\% 12,9\%-44,4\%). En cinco pacientes ingresados a la UCI se decidió limitar el esfuerzo terapéutico.

En total, 28 pacientes (25\% IC 95\% 17,3\%-34,1\%) fallecieron durante el seguimiento. De éstos, veinte $(71,4 \%)$ habían recibido una orden de limitación del esfuerzo terapéutico, 15 de las cuales ocurrieron antes de su ingreso a la UCl.

Al contrastar índices clínicos y de laboratorio entre supervivientes y fallecidos se apreciaron varias diferencias que se resumen en la Tabla 1 . En lo clínico, los pacientes fallecidos tendieron a ser mayores $(57,3 \pm 16,1$ vs 81,7 $\pm 10,6$ años, $p<$ $0,001)$, tuvieron menos días de clínica (mediana 6,5 días, RIC 3,5-10 días vs 3,0 días, RIC 0,5-6,5 días, $p<0,01$ ) y mostraron una mayor carga de comorbilidades (Índice de Charlson mediana de 2, RIC 0-3 puntos vs 6, RIC 3,5-6 puntos $\mathrm{p}<0,001)$, destacando una mayor frecuencia de hipertensos $(33,7 \%$ vs 
$71,4 \% p=0,001)$ y pacientes con insuficiencia cardiaca $(4,9 \%$ vs $21,4 \%, p=0,02$ ) entre los fallecidos. El laboratorio también mostró diferencias importantes entre grupos. Los fallecidos ingresaron con menores cifras de Pa/Fi (332 \pm 102 vs $209 \pm 104$, $\mathrm{p}<0,001)$, mayores alzas de proteína $C$ reactiva $(75,2 \pm 72,3$ vs $136,5 \pm 91,1 \mathrm{mg} / \mathrm{Lt}, \mathrm{p}<0,001$ ), lactato deshidrogenasa (306 \pm 132 vs $427 \pm 205, p=0,002)$ y uremia $(39,6 \pm 36$ vs 86,4 $\pm 59,5 \mathrm{mg} / \mathrm{dL}$ ). Al comparar las características del hemograma entre grupos se apreció que los fallecidos presentaron mayores recuentos leucocitarios totales $(6.920 \pm 2.540$ vs $9.300 \pm 3.800$ cels $\left./ \mathrm{mm}^{3}, \mathrm{p}<0,001\right)$ y de neutrófilos al ingreso $(4.710 \pm 2.280$ vs $7.900 \pm 3.600$ cels $\left./ \mathrm{mm}^{3}, p<0,001\right)$, mientras que el recuento linfocitario fue significativamente menor entre quienes fallecieron ( $1.510 \pm 1.155$ vs $855 \pm 370$ cels $\left./ \mathrm{mm}^{3}, p<0,001\right)$. Consecuentemente, al contrastar la relación neutrófilo a linfocito entre grupos se apreciaron diferencias significativas entre grupos, siendo mayor entre los fallecidos $(4,6 \pm 4,0$ vs 11,5 \pm $8,5, p<0,001)$. No se apreciaron contrastes significativos en ferritina de ingreso $(1.173 \pm 1.432$ vs $2.325 \pm 4.395, p=0,07)$, lactacidemia ni niveles de electrolitos plasmáticos.

\section{Capacidad predictiva de índices de laboratorio}

Las pruebas de laboratorio seleccionadas demostraron una capacidad moderada a buena en la predicción de tanto la necesidad de ventilación mecánica invasiva como mortalidad intrahospitalaria. Para el desenlace de necesidad de conexión a ventilación mecánica, los mejores índices se observaron en los valores de proteína C reactiva (AUC: 0,75, IC 95\% 0,65-0,86) y lactato deshidrogenasa (AUC 0,75, IC 95\% 0,61-0,90), sin que se apreciara una diferencia significativa entre ellas $(p=0,89)$. El recuento linfocitario y la relación neutrófilo a linfocito tuvieron desempeños inferiores para este último evento, con un AUC de 0,66 (IC 95\% 0,54-0,78) y 0,70 (IC 95\% 0,57-0,81), sin encontrarse diferencias estadísticamente significativas entre ellas ( $p=$ $0,53)$. Se muestra la comparación de estas últimas pruebas en la Figura 1.

En cuanto a la predicción de mortalidad, la relación neutrófilo a linfocito demostró tener la mejor capacidad predictiva de los parámetros seleccionados, con una AUC de 0,83 (0,75-0,91) para detectar pacientes en riesgo de fallecer. Al comparar esta evaluación con el recuento linfocitario absoluto y la proteína $C$ reactiva se apreciaron contrastes que alcanzaron significancia estadística por el método de Hanley y McNeal $(p=0,048)$. Se muestran los resultados de esta comparación en la Figura 2. Un valor de corte en la relación neutrófilo a linfocito de 5,5 o superior tuvo una sensibilidad del $80,8 \%$ y una especificidad del $70,7 \%$ para detectar pacientes que fueran a fallecer durante la estadía hospitalaria. De similar manera, al usar esta cifra como un factor de riesgo de mortalidad en análisis de supervivencia, se apreció un HR de mortalidad de 2,89 (IC 95\% 1,14-7,3, p = $0,025)$. Se muestra un resumen de las capacidades predictivas de las distintas pruebas seleccionadas en la Tabla 2, así como un análisis en curvas de supervivencia de Kaplan y Meier en la Figura 3.

\section{Discusión}

La pandemia actual por SARS-CoV-2 se ha caracterizado por una gran cantidad de pacientes que han requerido ingreso

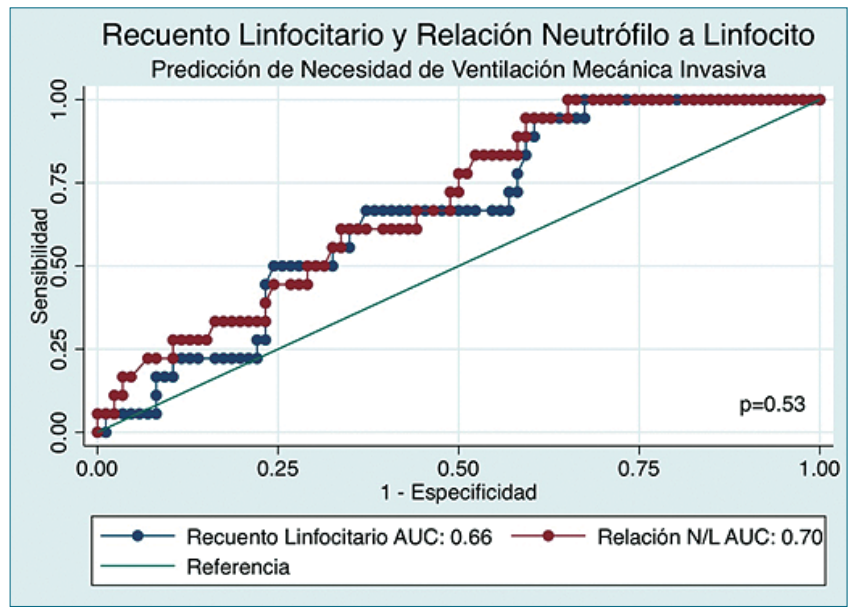

Figura 1.

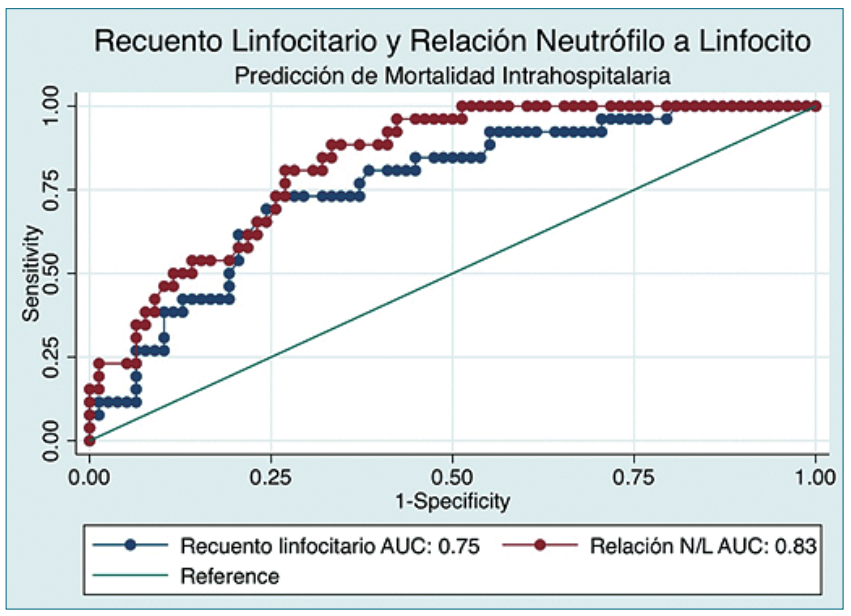

Figura 2.

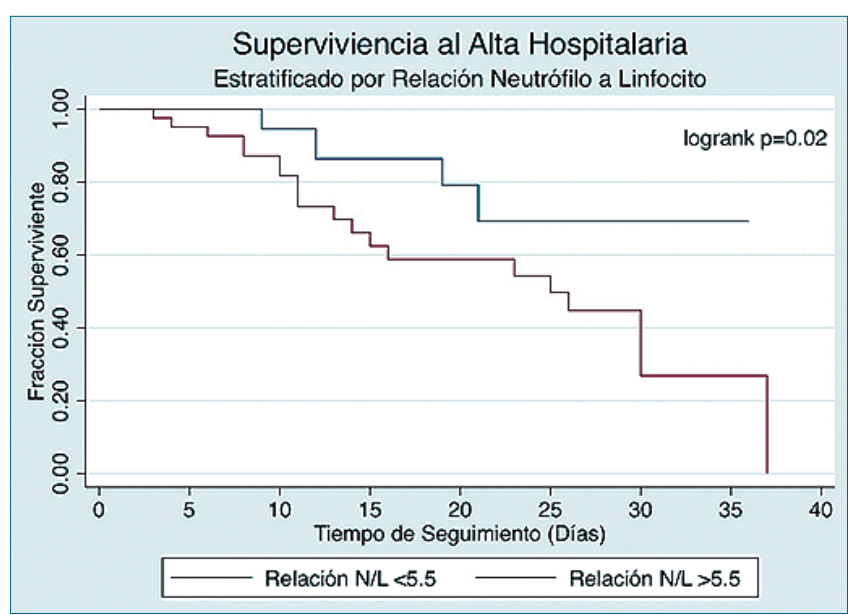

Figura 3. 
Tabla 2. Capacidad predictiva de índices de laboratorio

\begin{tabular}{|c|c|c|c|c|}
\hline Característica de laboratorio & Área bajo la curva (IC 95\%) & Punto de corte & Sensibilidad & Especificidad \\
\hline \multicolumn{5}{|c|}{ Predicción de necesidad de ventilación invasiva } \\
\hline Recuento linfocitario (cels/mm³) & $\begin{array}{c}0,66 \\
(0,54-0,78)\end{array}$ & 1.000 & $55,6 \%$ & $65,5 \%$ \\
\hline Relación neutrófilo a linfocito & $\begin{array}{c}0,70 \\
(0,57-0,81)\end{array}$ & 4,7 & $66,7 \%$ & $55,9 \%$ \\
\hline Proteína C reactiva (mg/Lt) & $\begin{array}{c}0,75 \\
(0,65-0,86)\end{array}$ & 100 & $72,2 \%$ & $70,9 \%$ \\
\hline Lactato deshidrogenasa (UI/L) & $\begin{array}{c}0,75 \\
(0,61-0,90)\end{array}$ & 280 & $80,0 \%$ & $53,3 \%$ \\
\hline Ferritina sérica (ug/dL) & $\begin{array}{c}0,75 \\
(0,61-0,91)\end{array}$ & 1.100 & $71,4 \%$ & $77,5 \%$ \\
\hline \multicolumn{5}{|c|}{ Predicción de mortalidad hospitalaria } \\
\hline Recuento linfocitario (cels/mm³) & $\begin{array}{c}0,75 \\
(0,65-0,86)\end{array}$ & 1.100 & $80,7 \%$ & $60,8 \%$ \\
\hline Relación neutrófilo a linfocito & $\begin{array}{c}0,83 \\
(0,75-0,91)\end{array}$ & 5,5 & $80,8 \%$ & $73,1 \%$ \\
\hline Proteína C reactiva (mg/Lt) & $\begin{array}{c}0,70 \\
(0,58-0,82)\end{array}$ & 85 & $76,0 \%$ & $62,0 \%$ \\
\hline Lactato deshidrogenasa (UI/L) & $\begin{array}{c}0,69 \\
(0,54-0,83)\end{array}$ & 310 & $70,0 \%$ & $62,9 \%$ \\
\hline Ferritina sérica (ug/dL) & $\begin{array}{c}0,58 \\
(0,43-0,73)\end{array}$ & 770 & $63,2 \%$ & $59,1 \%$ \\
\hline
\end{tabular}

a unidades de paciente crítico, llevando a los sistemas de salud al límite de su capacidad. Es por esto que cada vez se hace más necesaria la identifiación de factores de riesgo que permitan clasificar mejor a aquellos sujetos que pudiesen evolucionar a una enfermedad grave. En el presente estudio, se reporta que una relación neutrófilo a linfocito superior a 5,5 tuvo una favorable capacidad diagnóstica para identificar pacientes que tendrán una evolución tórpida, incluyendo desenlaces como necesidad de ventilación mecánica invasiva y mortalidad.

\section{Recuento linfocitario y relación neutrófilo a linfocito en SARS-CoV-2}

A la fecha, la mayoría de los estudios publicados hacen referencia a los biomarcadores encontrados en no-sobrevivientes[19]-[21]. Los hallazgos típicos de laboratorio reportados incluyen la elevación de la proteína $C$ reactiva y lactato deshidrogenasa, alza de transaminasas y linfopenia (recuento absoluto de linfocitos $<1.000$ células/uL)[22]. No obstante, pocos trabajos hacen referencia a la capacidad predictiva de dichos hallazgos, dificultando su implementación en la práctica clínica habitual. Los resultados presentados en este trabajo son similares a los reportados por Chuan Qin et al., quien en un estudio retrospectivo de 452 pacientes, describe una relación neutrófilo a linfocito elevada en los casos severos comparado con el grupo no-severo (5,5 vs 3,2; $<<0,001$ )[23]. En otro estudio orientado a la capacidad pronóstica de la linfopenia aislada, Li Tan et al., a través de un modelo basado en el análisis del porcentaje de linfocitos durante la hospitalización, reporta una apropiada capacidad para clasificar y predecir el pronóstico. En este reporte, los pacientes que tuvieron más de $20 \%$ de linfocitos entre los días
10 a 12 de síntomas tuvieron una evolución favorable, mientras que quienes presentaron tuvieron cifras menores y sostenidas en el tiempo tuvieron un curso severo asociado a mayor mortalidad[24].

La etiología tras de la linfopenia en pacientes infectados con SARS-CoV-2 no está del todo dilucidada. Este fenómeno también ha sido descrito con otras infecciones virales, incluyendo otros virus causantes del SARS, virus Ébola y virus respiratorio sincicial[25]. Existen varios factores que pudiesen contribuir en este fenómeno. En primer lugar, debe considerarse a la respuesta inflamatoria de los pacientes. Al iniciarse la respuesta inflamatoria secundaria a la infección por SARS-CoV-2 se producen elevaciones de citokinas como el factor de necrosis tumoral alfa (TNF- $\alpha$ ) interleukina (IL) $1 \alpha$, IL-1 $\beta$, interferon- $\gamma$, IL-2, IL-3, IL-5, IL-6, IL-8 e IL-12, entre otras, la que posteriormente será modulada por otros mediadores[1],[25]. Esta respuesta antiinflamatoria es orquestada en base a cortisol y otras interleukinas, incluyendo IL-4, IL-10 e IL-13. El rol del cortisol resulta especialmente interesante por su capacidad de inducir tanto remoción de la circulación periférica como apoptosis linfocitaria, la que incluso ha mostrado correlaciones fisiológicas con el ciclo circadiano de secreción de esta hormona. Resulta además interesante considerar que así como los corticoides inducen apoptosis de linfocitos, también causan inhibición de estos procesos en neutrófilos[25]-[27]. De esta manera, es posible postular que la relación neutrófilo a linfocito pudiera reflejar perfiles de liberación de citoquinas entre pacientes con enfermedad más grave. Esto también podría explicar la razón detrás de la mejoría en capacidad diagnóstica que se observa en relación a la utilización del recuento linfocitario como predictor aislado, si bien 
no se alcanza significancia estadística en esta cohorte. Por otra parte, también se ha demostrado que los llinfocitos expresan el receptor ACE2 en su membrana, por lo que pudiesen ser infectados por el virus gatillando la muerte celular en forma directa.

\section{Otros mediadores}

En un estudio de Guan et al., se demostró una elevación en la proteína $C$ reactiva (PCR) de 60,7\% entre los pacientes con enfermedad por COVID-19[17]. Al analizar los casos más severos, se observó un marcado incremento comparado con los casos no severos (81,5\% vs 56,4\% ( $p<0,001)[10]$. El ingreso a $\mathrm{UCl}$, uso de ventilación mecánica y muerte, también se asoció a valores más elevados de PCR. En otro estudio, Deng et al., observó valores de PCR significativamente mayores entre los casos fatales $(109,25 \mathrm{mg} / \mathrm{L}$ vs 3,22 mg/L, $p<0,001)$. En nuestro estudio encontramos una media de PCR superior a la descrita $(90,0$ $\mathrm{mg} / \mathrm{L})$, siendo incluso mayor en los fallecidos (136,5 mg/dL). La capacidad predictiva fue similar a la observada en otros índices de laboratorio, probablemente por la estrecha vinculación de la PCR con la liberación de citoquinas proinflamatorias. Similares hallazgos fueron observados con LDH, los que también han sido reportados previamente en la literatura. Zhou et al. y Wu et al., describieron mayores valores de LDH entre fallecidos[28],[29]. Sin embargo, el primer estudio encontró que estos valores disminuyeron al día 13 entre pacientes supervivientes. En nuestro estudio, también encontramos valores de LDH más altos entre los fallecidos, sin embargo, no se analizó la evolución en días posteriores de enfermedad. Wu et al., mostró que valores elevados de lactato deshidrogenasa se asociaron a mayor desarrollo de Síndrome de Distress Respiratorio Agudo. En el presente estudio, tanto PCR como LDH mostraron los mejores índices de predicción respecto a la probabilidad de conexión a ventilación mecánica.

\section{Limitaciones del estudio}

Si bien el presente es un estudio de cohorte prospectiva con una muestra variada de individuos con infección a SARSCoV-2, existen algunas limitantes que deben ser consideradas. En primer lugar, este trabajo evalúa a la relación neutrófilo a linfocito como predictor único, sin considerar ajustes por otros factores o morbilidades que pudieran impactar el pronóstico de estos pacientes. Haber realizar un análisis multivariante para purificar de mejor manera la asociación hubiera sido ideal para afiatar el rol de esta prueba. No obstante, dicho análisis hubiera exigido un número de participantes ostensiblemente mayor, el cual hubiera retrasado importantemente la realización del estudio en un escenario de pandemia en el que contar con información para guiar esfuerzos clínicos resulta fundamental. El tamaño muestral obtenido permitió de todas maneras obtener una precisión aceptable para el indicador analizado, el que puede de todas formas sumarse a las herramientas disponibles para el clínico en la evaluación de estos pacientes. Una segunda consideración está en que las mediciones se efectuaron una sola vez entre los participantes incluidos, sin tenerse información evolutiva de las alteraciones durante la hospitalización, lo que hubiera además facilitado la comparabilidad con otras experiencias. No obstante, también debe tenerse en cuenta que el objetivo de este trabajo es contar con información precoz en el curso de la enfermedad para orientar la toma de decisiones clínicas. El perfil evolutivo de los estudios de laboratorio es en este sentido es interesante, pero dificulta la aplicación práctica de los hallazgos en la evaluación de riesgo de pacientes individuales al requerir más tiempo y mediciones para su estimación. Otra limitante relevante está en que se analizó el recuento total de linfocitos, sin considerar subpoblaciones o mediciones de citokinas específicas que permitieran dilucidar de mejor manera las razones detrás de la linfopenia en COVID-19. Si bien contar con esta información resulta sin duda interesante para dilucidar la fisiopatología de este fenómeno, estas mediciones adicionales no se encuentran en sintonía con el objetivo fundamental del estudio, que es evaluar un marcador sencillo y ampliamente disponible para evaluar la gravedad de pacientes con infecciones a SARS-CoV-2. Estudios futuros orientados a establecer pronóstico en pacientes con COVID-19 deben considerar estas limitaciones para ahondar más profundamente en el establecimiento de herramientas pronósticas en la enfermedad, idealmente realizando estudios multicéntricos que permitan alcanzar un tamaño muestral suficiente para la construcción de índices multivariados.

\section{Síntesis}

Una relación neutrófilo a linfocito superior a 5,5 demostró ser un factor de riesgo de eventos clínicos adversos en esta cohorte prospectiva. Su capacidad diagnóstica como predictor único fue favorable, por lo que su utilización puede ser considerada dentro del armamentario diagnóstico de clínicos que busquen establecer el pronóstico de pacientes con COVID-19.

\section{Referencias}

1. Wiersinga WJ, rhodes $A$, Cheng AC, Peacock SJ, Prescott HC. Pathophysiology, Transmission, Diagnosis, and Treatment of Co- ronavirus Disease 2019 (COVID-19): A review. JAMA [Internet]. 2020 Aug 25;324(8):782-93. doj: https://doi.org/10.1001/ jama.2020.12839.

2. Zhu N, Zhang D, Wang W, Li X, Yang B, Song J, et al.; China Novel Coronavirus Investigating and Research Team. A novel coronavirus from patients with pneumonia in China, 2019. N Engl J Med. 2020 Feb;382(8):727-33. https://doi.org/10.1056/ NEJMoa2001017 PMID:31978945

3. Huang I, Pranata r. Lymphopenja in severe coronavirus disease-2019 (COVID-19): Systematic review and meta-analysis. J Intensive Care. 2020;8(1):1-10. https://doi.org/10.1186/s40560020-00453-4.

4. Yuki K, Fujiogi M, Koutsogiannaki S. COVID-19 pathophysjology: A review. Clin Immunol. 2020;215(April). https://doi.org10.1016/j.clim.2020.108427

5. Murthy S, Gomersall CD, Fowler rA. Care for Critically III Patients With COVID-19. JAMA [Internet]. 2020 Mar 11;1-2. https://doi.org/10.1001/jama.2020.3633..

6. Berlin DA, Gulick rM, Martinez FJ. Severe Covid-19. N Engl J Med. 2020;1-10. https://doi.org/10.1056/NEJMcp2009575.

7. Tan L, Wang Q, Zhang D, Ding J, Huang Q, Tang YQ, et al. Lym- phopenja predicts disease severity of COVID-19: a descriptive and predictive study. Signal Transduct Target Ther. 2020;5(1):16-8. https://doi.org/10.1038/s41392-020-0148-4.

8. Huang $C$, Wang $Y, L_{i} X$, ren $L$, Zhao J, Hu Y, et al. Clinjical featu- res of patients infected $w_{j}$ th 2019 novel coronavirus in 
Wuhan, China. Lancet. 2020;395(10223):497-506. https://doi. org/10.1016/S0140-6736(20)30183-5.

9. Richardson S, Hirsch JS, Narasimhan M, Crawford JM, McGinn T, Davidson KW, et al.; the Northwell COVID-19 Research Consortium. Presenting Characteristics, Comorbidities, and Outcomes Among 5700 Patients Hospitalized With COVID-19 in the New York City Area. JAMA. 2020 May;323(20):2052-9. https://doi. org/10.1001/jama.2020.6775 PMID:32320003

10. Guan WJ, Ni ZY, Hu Y, Liang WH, Ou CQ, He JX, et al.; China Medical Treatment Expert Group for Covid-19. Clinical Characteristics of Coronavirus Disease 2019 in China [Internet]. N Engl J Med. 2020 Apr;382(18):1708-20. https://doi.org/10.1056/NEJMoa2002032 PMID:32109013

11. von Elm E, Altman DG, Egger M, Pocock SJ. G0tzsche PC, Vandenbroucke JP. The Strengthening the reporting of Observational Studies in Epidemiology (STrOBE) statement: guidelines for reporting observational studies [Internet]. J Clin Epidemiol. 2008 Apr;61(4):344-9. https://doi.org/10.1016/j.jclinepi.2007.11.008 PMID:18313558

12. Charlson M, Szatrowski TP, Peterson J, Gold J. Validation of a combined comorbidity index [Internet]. J Clin Epidemiol. 1994 Nov;47(11):1245-51. https://doi.org/10.1016/08954356(94)90129-5 PMID:7722560

13. Hall WH. ramachandran $r$, Narayan $S$, Jani $A B$, Vijayakumar $\mathrm{S}$. An electronic application for rapidly calculating Charlson comor- bidity score. BMC Cancer. 2004;4(1):94. https://doi. org/10.1186/1471-2407-4-94 PMID:15610554

14. Singer M, Deutschman CS, Seymour CW, Shankar-Hari M, Annane D, Bauer M, et al. The Third International Consensus Definitions for Sepsis and Septic Shock (Sepsis-3) [Internet]. JAMA. 2016 Feb;315(8):801-10. https://doi.org/10.1001/ jama.2016.0287 PMID:26903338

15. Zhang D, Fu R, Li Y, Li H, Li Y, Li H. Comparison of the clinical characteristics and prognosis of primary versus secondary acute gastrointestinal injury in critically ill patients. J Intensive Care. 2017 Apr;5(1):26. https://doi.org/10.1186/s40560-017-0221-4 PMID:28435684

16. de Grooth HJ, Geenen IL, Girbes AR, Vincent JL, Parienti JJ, Oudemans-van Straaten HM. SOFA and mortality endpoints in randomized controlled trials: a systematic review and meta-regression analysis [Internet]. Crit Care. 2017 Feb;21(1):38. https:// doi.org/10.1186/s13054-017-1609-1 PMID:28231816

17. Terpos E, Ntanasis-Stathopoulos I, Elalamy I, Kastritis E, Sergentanis TN, Politou M, et al. Hematological findings and complications of COVID-19. Am J Hematol. 2020 Jul;95(7):834-47. https://doi. org/10.1002/ajh.25829 PMID:32282949

18. Wang D, Hu B, Hu C, Zhu F, Liu X, Zhang J, et al. Clinical Characteristics of 138 Hospitalized Patients With 2019 Novel Coronavirus-Infected Pneumonia in Wuhan, China [Internet]. JAMA. 2020 Mar;323(11):1061-9. https://doi.org/10.1001/jama.2020.1585 PMID:32031570

19. Yang $X, Y u Y, X u$ J, Shu H, Xia J, Liu H, et al. Clinical course and outcomes of critically ill patients with SARS-CoV-2 pneumonia in Wuhan, China: a single-centered, retrospective, observational study. Lancet Respir Med. 2020 May;8(5):475-81. https://doi. org/10.1016/S2213-2600(20)30079-5 PMID:32105632

20. Deng Y, Liu W, Liu K, Fang YY, Shang J, Zhou L, et al. Clinical characteristics of fatal and recovered cases of coronavirus disease 2019 in Wuhan, China: a retrospective study. Chin Med J (Engl). 2020 Jun;133(11):1261-7. https://doi.org/10.1097/ CM9.0000000000000824 PMID:32209890

21. Ruan Q, Yang K, Wang W, Jiang L, Song J. Clinical predictors of mortality due to COVID-19 based on an analysis of data of 150 patients from Wuhan, China [Internet]. Intensive Care Med. 2020 May;46(5):846-8. https://doi.org/10.1007/s00134-020-05991-x PMID:32125452

22. Rodríguez-Morales AJ, Cardona-Ospina JA, Gutiérrez-Ocampo E, Villamizar-Peña r, Holguin-rivera Y, Escalera-Antezana JP, et al. Clinical, laboratory and imaging features of COVID-19: A syste- matic review and meta-analysis. Travel Med Infect Dis [Internet]. 2020;34(March):101623. https://doi.org/10.1016/j. tmaid.2020.101623.

23. Qin C, Zhou L, Hu Z, Zhang S, Yang S, Tao Y, et al. Dysregulation of Immune Response in Patients With Coronavirus 2019 (COVID-19) in Wuhan, China [Internet]. Clin Infect Dis. 2020 Jul;71(15):762-8. https://doi.org/10.1093/cid/ciaa248 PMID:32161940

24. Tan L, Wang Q, Zhang D, Ding J, Huang Q, Tang YQ, et al. Lymphopenia predicts disease severity of COVID-19: a descriptive and predictive study [Internet]. Signal Transduct Target Ther. 2020 Mar;5(1):33. https://doi.org/10.1038/s41392-020-0148-4 PMID:32296069

25. Panesar NS. What caused lymphopenia in SArS and how reliable is the lymphokine status in glucocorticoid-treated pa- tients? Med Hypotheses. 2008;71(2):298-301. https://doi.org/10.1016/j. mehy.2008.03.019

26. Fauci AS, Murakami T, Brandon DD, Loriaux DL, Lipsett MB Mechanisms of corticosteroid action on lymphocyte subpopu- lations. Cell Immunol. 1980;49(1):43-50. https://doi. org/10.1016/0008-8749(80)90054-4 PMID:6965362

27. Herold MJ, McPherson KG, Reichardt HM. Glucocorticoids in T cell apoptosis and function. Cell Mol Life Sci. 2006 Jan;63(1):60-72. https://doi.org/10.1007/s00018-005-5390-y PMID: 16314919

28. Wu C, Chen X, Cai Y, Xia J, Zhou X, Xu S, et al. Risk Factors Associated With Acute Respiratory Distress Syndrome and Death in Patients With Coronavirus Disease 2019 Pneumonia in Wuhan, China. JAMA Intern Med. 2020 Jul;180(7):934-43. https://doi. org/10.1001/jamainternmed.2020.0994 PMID:32167524

29. Zhou F, Yu T, Du R, Fan G, Liu Y, Liu Z, et al. Clinical course and risk factors for mortality of adult inpatients with COVID-19 in Wuhan, China: a retrospective cohort study [Internet]. Lancet. 2020 Mar;395(10229):1054-62. https://doi.org/10.1016/S01406736(20)30566-3 PMID:32171076 